\title{
Sarcoidosis with splenic involvement diagnosed via endoscopic ultrasound-guided fine-needle biopsy
}

\author{
Hyung Ku Chon ${ }^{1}$, Keum Ha Choi ${ }^{2}$, and Tae Hyeon Kim ${ }^{1}$
}

'Division of Gastroenterology, Department of Internal Medicine, ${ }^{2}$ Department of Pathology, Wonkwang University Hospital, Iksan, Korea
Received: November 25, 2016 Revised : December 22, 2016 Accepted: January 2, 2017

\section{Correspondence to}

Tae Hyeon Kim, M.D.

Tel: +82-63-859-2564

Fax: $+82-63-855-2025$

E-mail:jipsinsa@naver.com
A 48-year-old woman presented with dyspepsia, a 5-kg weight loss, and night sweating for 2 months. She had no underlying disease and denied alcohol consumption or smoking. Her vital signs, physical examination, and laboratory results were unremarkable. Abdominal computed tomography (CT) showed multiple low-density lesions of variable sizes in the spleen, with perisplenic lymph node enlargement (Fig. 1A). Chest CT revealed enlargement
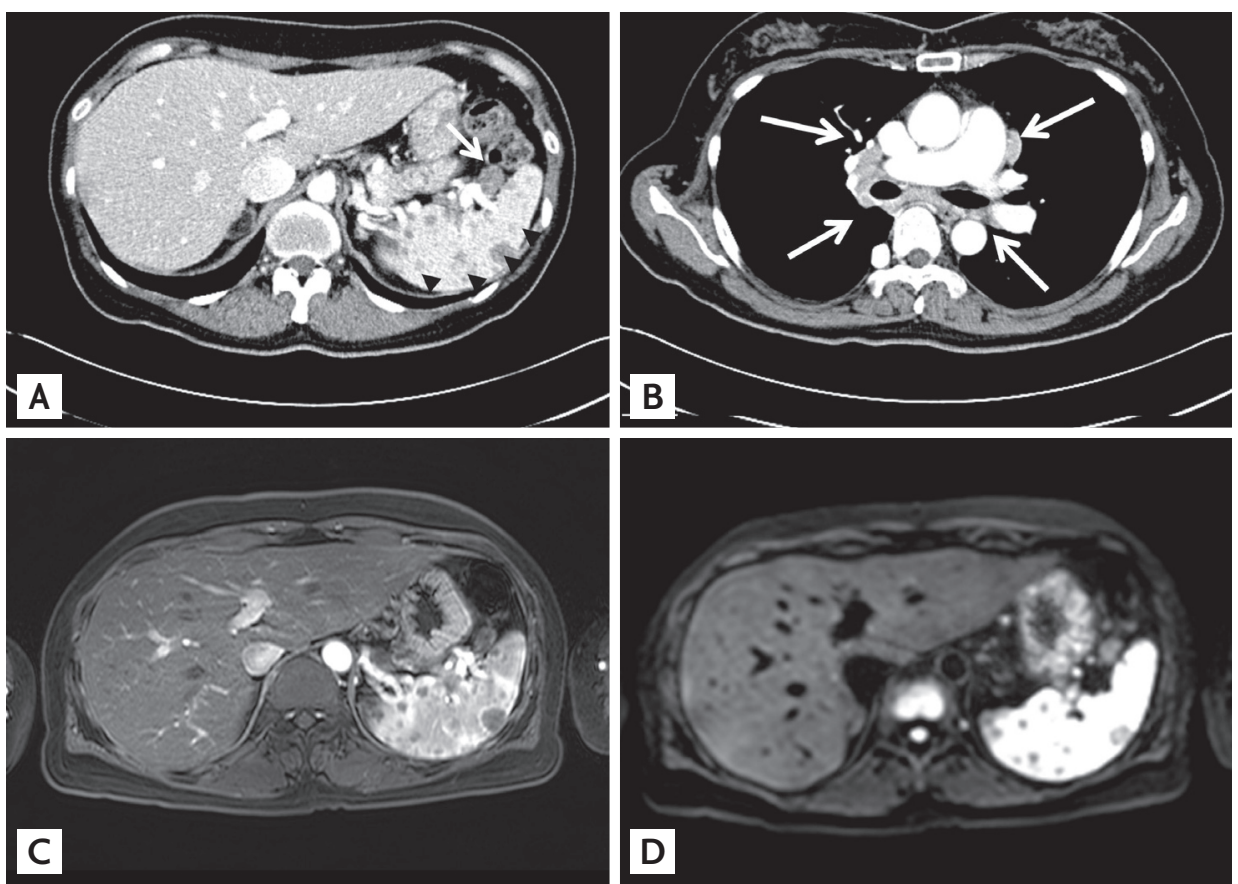

Figure 1. (A) Transverse abdominal computed tomography (CT) showing multiple ill-defined low-attenuation splenic lesions (black arrowheads) with lymphadenopathy in the splenic hilum (white arrow). (B) Axial CT shows enlargement in the bilateral hilar, subcarinal, and subaortic lymph nodes (white arrows) without central necrosis or lung parenchymal abnormalities. (C) T2-weighted images showing variable hypoechoic nodules in the spleen. (D) Multiple lesions presenting low signal intensity in diffusion-weighted images. 

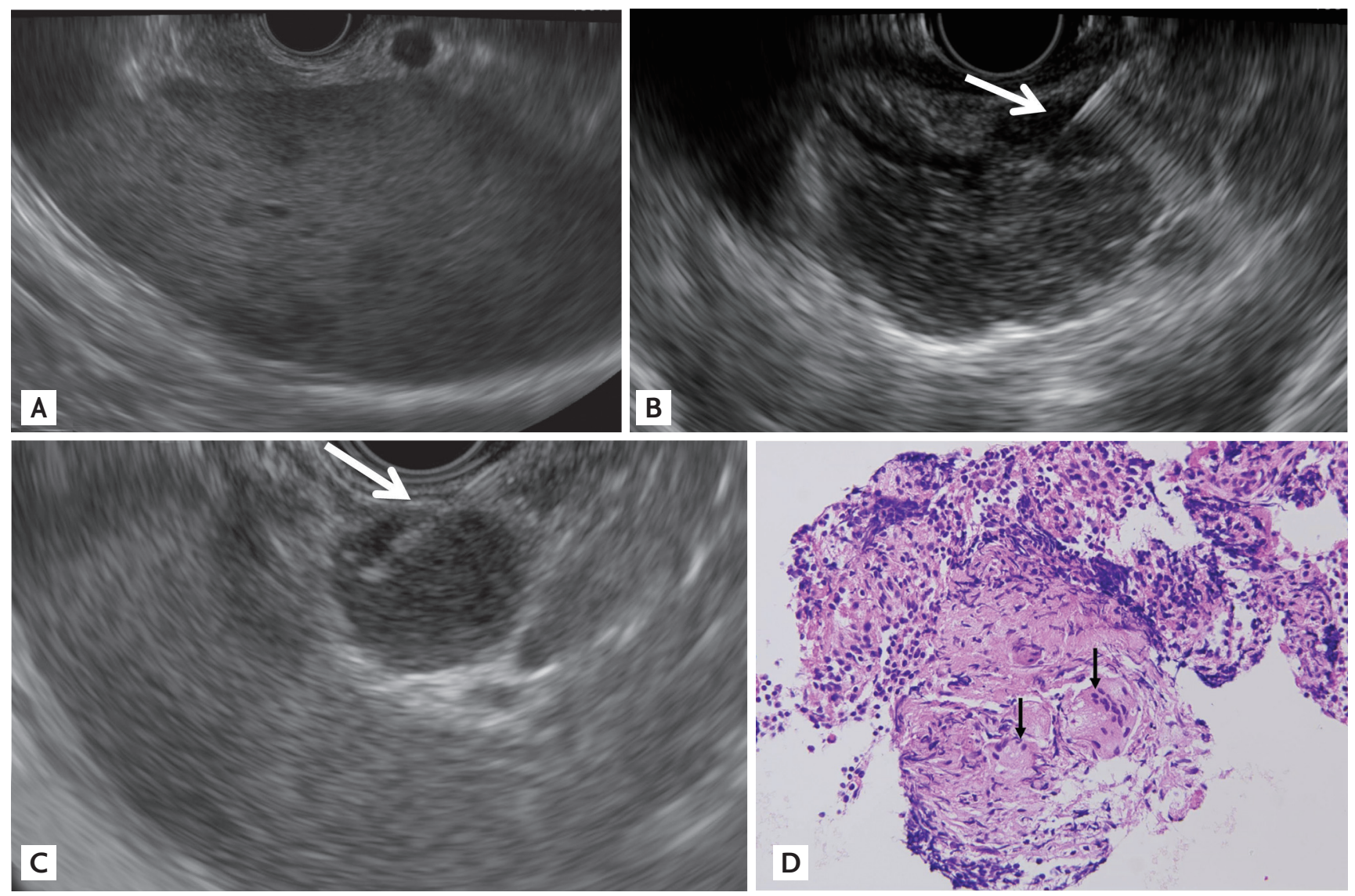

Figure 2. (A) Endoscopic ultrasound (EUS) image showing multiple variable, ill-defined, hypoechoic splenic lesions. EUS-fine needle biopsy with a 22-G ProCore needle (white arrow) was performed for the subcarinal (B) and perisplenic (C) lymph nodes. (D) High-power view showing a noncaseating granuloma composed of epithelioid histiocytes and Langhans multinucleated giant cells (arrows) (H\&E, ×400).

lesions were seen (Fig. 2A), and EUS-guided fine-needle biopsy (FNB) with a 22-gauge needle (Echotip ProCore, Cook Endoscopy Inc., Limerick, Ireland), targeting the lymph nodes of the subcarinal and perisplenic regions, was performed (Fig. 2B and 2 C). Pathologic examination showed noncaseating epithelioid cell granulomas with multinucleated giant cells (Fig. 2D). The results of the polymerase chain reaction for Mycobacterium tuberculosis and special stains for acid-fast bacilli and fungi in tissue samples were negative. The findings indicated sarcoidosis with splenic involvement.

Sarcoidosis with splenic nodular lesions is uncommon and difficult to diagnose in the absence of clinical suspicion. EUS-FNB for sarcoidosis is a minimally invasive technique, enabling a more accurate tissue acquisition under real-time visualization, avoiding cutaneous infection, and allowing evaluation of lymph nodes measuring $<1 \mathrm{~cm}$, compared with transbronchial lung biopsy, mediastinoscopy, or percutaneous biopsy.

Unlike conventional needles, ProCore needles have a reverse bevel at the tip, which promotes the collection of core tissue samples, enabling a definitive diagnosis of malignant or benign conditions.

When a clinician encounters a patient with multiple enlarged lymph nodes and splenic nodular lesions, sarcoidosis should be considered in the differential diagnosis and EUS-FNB may be a useful diagnostic modality.

\section{Conflict of interest}

No potential conflict of interest relevant to this article was reported.

\section{Acknowledgments}

This study was supported by the Wonkwang University fund in 2017 . 